\title{
SISTEMA REPRODUCTIVO Y POLINIZACIÓN DE ESPECIES LEÑOSAS EN UNA SELVA SUBTROPICAL INVADIDA POR PLANTAS EXÓTICAS
}

\author{
NORBERTO H. MONTALDO ${ }^{1}$, ANITA I. MANTESE ${ }^{1}$ y G. GERMÁN ROITMAN²
}

\begin{abstract}
Summary: Breeding system and pollination of woody species in a subtropical forest invaded by alien plants. In the riparian forest of Punta Lara (Buenos Aires province, Argentina) seven woody bird-dispersed species coexist, five of which are native (Ocotea acutifolia, Allophylus edulis, Myrsine laetevirens, Blepharocalyx salicifolius and Citharexylum montevidense), and two are invasive aliens (Rubus ulmifolius and Ligustrum lucidum). While seed dispersal of these species has been studied, other aspects of their reproduction are unknown. In this paper we studied the breeding systems, the quality of the pollination service they receive and their floral visitors. All species are xenogamous. One species ( $M$. laetevirens) is anemophilous and the others are visited mostly by Diptera, Hymenoptera and Lepidoptera. For most of the native species the pollination service seems insufficient. In $A$. edulis and $B$. salicifolius natural fruit set was lower than that of hand cross-pollinated flowers, only $O$. acutifolia and the two exotic showed stigmatic pollen loads sufficient to achieve maximum seed production. A possible explanation for the patterns found lies in the reduction of populations of native species due to the expansion of the aliens, particularly L. Iucidum.
\end{abstract}

Key words: Invasive plants, riparian forest, reproductive biology, pollination success, stigmatic pollen loads, Argentina.

\begin{abstract}
Resumen: En la selva ribereña de Punta Lara (provincia de Buenos Aires, Argentina) conviven siete especies leñosas dispersadas por aves, de las cuales cinco son nativas (Ocotea acutifolia, Allophylus edulis, Myrsine laetevirens, Blepharocalyx salicifolius y Citharexylum montevidense) y dos son exóticas invasoras (Rubus ulmifolius y Ligustrum lucidum). Si bien allí se ha estudiado la dispersión de semillas de estas especies, otros aspectos de su reproducción son desconocidos. En este trabajo estudiamos su sistema reproductivo, la calidad del servicio de polinización que reciben y los visitantes florales. Todas las especies son xenógamas. Una especie (M. laetevirens) es anemófila y las demás son visitadas principalmente de Dípteros, Himenópteros y Lepidópteros. El servicio de polinización parece insuficiente en la mayoría de las especies nativas. $A$. edulis y $B$. salicifolius produjeron menos frutos en condiciones naturales que cuando se polinizó las flores con polen de otro individuo y sólo O. acutifolia y las dos especies exóticas mostraron cargas polínicas estigmáticas suficientes para alcanzar el máximo de producción de semillas. Una posible explicación de los patrones encontrados podría ser la reducción de las poblaciones de las especies nativas producida por la expansión de las exóticas, en particular de $L$. lucidum.
\end{abstract}

Palabras clave: Plantas invasoras, selva ribereña, biología reproductiva, éxito de la polinización, cargas de polen estigmáticas, Argentina.

\section{INTRODUCCIÓN}

En las plantas con flores la disponibilidad de polen suficiente es esencial para la perpetuación de las poblaciones en el largo término, ya que

Cátedras de Botánica General ${ }^{1}$ y de Jardinería ${ }^{2}$, Facultad de Agronomía, Universidad de Buenos Aires, Av. San Martín 4453, (C1417DSE), Buenos Aires, Argentina. E-mail: montaldo@agro.uba.ar afecta directamente la producción de semillas y la variabilidad genética (Ashman et al., 2004; Bell et al., 2005; Matsumoto et al., 2011). La imposibilidad de recibir polen de otro individuo disminuiría la producción de semillas (si son especies autoincompatibles) o podría implicar mayor tasa de autopolinización y aumento de la depresión por endogamia (si son auto-compatibles) (Spira, 2001). El fracaso del proceso de polinización puede conducir a la extinción (Bond, 1994). 
Los principales polinizadores son los animales (Ollerton et al., 2011) y muchas plantas dependen en forma obligada o facultativa de interacciones con animales para formar sus semillas (Burd, 1994). La relación mutualista planta-polinizador se encuentra amenazada por los disturbios humanos, entre ellos la introducción de especies exóticas (Kearns et al., 1998). Algunas de estas especies pueden experimentar un gran crecimiento poblacional e invadir el ecosistema, generando consecuencias devastadoras tanto ecológicas como económicas (Elton, 1958; Van Dyke, 2008). Las especies exóticas invasoras afectan la estructura, composición y funcionamiento de los ecosistemas invadidos de muchas maneras (Vitousek et al., 1997), alterando interacciones ecológicas que surgieron en escalas de tiempo evolutivo (Traveset \& Richardson, 2006, 2014). En el éxito de las especies nuevas influye su capacidad de interactuar con especies nativas y exóticas que faciliten su supervivencia, desarrollo y permanencia en el nuevo ambiente (e.g. Aizen, 2007). La limitación de polinizadores no parece ser un obstáculo importante para las plantas introducidas (Richardson et al., 2000), porque por lo común se integran bien a las redes nativas preexistentes (Memmott \& Waser, 2002; Morales \& Aizen, 2006; Aizen et al., 2008; Vila et al., 2009). Además el sistema reproductivo de las especies exóticas invasoras estaría fuertemente sesgado hacia la reproducción uniparental (a través de autocompatibilidad o apomixis, y capacidad de auto-polinización autónoma), lo cual asegura la reproducción y el establecimiento en un sitio nuevo (Rambuda \& Johnson, 2004; Hao et al., 2011).

En los estudios sobre interacciones de polinización entre plantas nativas y exóticas en general se confirma un efecto negativo de las exóticas sobre la reproducción de las nativas que florecen con ellas (Chittka \& Schürken, 2001; Morales \& Traveset, 2009). Sin embargo, no siempre se produce un efecto (Bjerknes et. al., 2007; Kaiser-Bunbury \& Müller, 2009; Traveset, 2015) y a veces éste puede resultar positivo al facilitar la polinización de las nativas (Rathcke, 1983; Memmott \& Waser, 2002; Bjerknes et. al., 2007; Traveset, 2015), dependiendo de la especie en cuestión (Moragues \& Traveset, 2005). No obstante, es claro que la colonización por exóticas puede introducir una distorsión en el ambiente polínico de la comunidad de plantas (Chittka \& Schürken,
2001) y en su interacción con los polinizadores (Aizen et al., 2008). Esta alteración se traduce en una modificación de la cantidad de visitas que recibe una flor (componente cuantitativo) y/o de la calidad de la polinización (el número de granos de polen conespecíficos depositados por visita), como resultado de competencia por polinizadores o modificaciones en la composición relativa de este gremio (Waser, 1983; Bell et al., 2005; Totland et al., 2006), entrega de menor cantidad de polen y/o de polen inadecuado (McLernon et al., 1996; Brown \& Mitchell, 2001, Matsumoto et al., 2010, 2011; Takakura \& Fujii, 2010), entre otros. Dicha interacción competitiva es más marcada en ambientes severos, donde las poblaciones de polinizadores son menores (Rathcke, 1983).

La selva ribereña de Punta Lara (provincia de Buenos Aires, Argentina) es el relicto de selva subtropical más austral del continente y representa un ecosistema empobrecido (Cabrera, 1960; para una hipótesis alternativa ver Guerrero, 2014). Por su fisionomía y composición florística es una prolongación de las selvas en galería del río Uruguay que van perdiendo especies a medida que aumenta la latitud. Por ejemplo hay más de 60 especies arbóreas en Misiones y poco más de 15 al llegar a la ribera del Río de la Plata (Cabrera, 1975). El desarrollo de esta selva en una región de clima templado es posible por la compensación de factores climáticos (microclima más húmedo y cálido que la región circundante) y edáficos (suelo aluvial muy húmedo) que realiza el Río de la Plata (Cabrera \& Dawson, 1944). Al encontrarse en situación de marginalidad ecológica es un sistema potencialmente vulnerable, porque el proceso de invasión depende de las propiedades de las especies invasoras (e.g. rápido crecimiento, gran producción de semillas y buena dispersión de las mismas, etc.) y de las características del ambiente a invadir (Noble, 1989).

Los hábitats ribereños como la selva de Punta Lara albergan un alto porcentaje de especies exóticas, ya que tradicionalmente han sido disturbados por actividades humanas que implican alta exposición a sus propágulos (Rejmánek, 1989). De hecho esta selva está invadida por plantas exóticas, Ligustrum lucidum Ait. ("ligustro", Oleaceae, originario de China) predomina en el estrato arbóreo y Rubus ulmifolius Schott ( "zarzamora", Rosaceae, nativa de la región Mediterránea) ocupa los claros de la selva. Ambas especies son dispersadas por aves frugívoras 


\section{N. H. Montaldo et al. - Polinización en una selva invadida por plantas exóticas}

(Montaldo, 1993, 2005) y tienen mayor producción y dispersión de diásporas que las plantas ornitócoras nativas del área (Montaldo, 2000).

El objetivo del trabajo fue estudiar el sistema reproductivo, el servicio de polinización (cantidad y calidad del polen que arriba al estigma) y los visitantes florales de especies nativas y exóticas que son dispersadas por aves frugívoras en la selva de Punta Lara. Trabajamos con este gremio porque comprende las plantas que predominan en la selva: las dos especies exóticas y 9 de las 16 especies nativas que integran el estrato arbóreo dependen de dicho agente (Montaldo, 2005) y porque la dispersión por aves frugívoras juega un papel muy relevante en el éxito de la invasión por plantas exóticas (Richardson et al., 2000; Traveset \& Richardson, 2014). Las aves son el agente de dispersión más importante para árboles y arbustos exóticos invasores (Richardson \& Rejmánek, 2011).

\section{Materiales y Métodos}

\section{Sitio del estudio y especies estudiadas}

Los trabajos de campo se llevaron a cabo en la Reserva Natural de Punta Lara $\left(34^{\circ} 47^{\prime} \mathrm{S}, 58^{\circ}\right.$ $01^{\prime} \mathrm{O}$ ), cuyo principal núcleo selvático ocupa aproximadamente 10 ha y se extiende desde la ribera del Río de la Plata, a ambos lados del arroyo Las Cañas. Una descripción del clima, suelo y de las comunidades vegetales de la reserva puede encontrarse en Cabrera \& Dawson (1944).

Se trabajó con 7 especies de plantas leñosas dispersadas por aves frugívoras: 2 exóticas (Rubus ulmifolius y Ligustrum lucidum) y 2 nativas con flores monoclinas (Blepharocalyx salicifolius (H.B.K.) Berg = anacahuita, Myrtaceae, y Citharexylum montevidense (Spreng.) Mold. = espina de bañado o tarumá, Verbenaceae) y 3 nativas dioicas (Ocotea acutifolia (Nees) Mez. = laurel, Lauraceae; Allophylus edulis (Camb.) Radlk. = chal chal, Sapindaceae, y Myrsine laetevirens (Mez.) Arechav. = canelón, Myrsinaceae). En todas, las flores pistiladas poseen estambres vestigiales.

\section{Sistema reproductivo}

Para el estudio del sistema reproductivo de las plantas, en el año 2000 se eligieron individuos de las distintas especies y se hicieron pruebas preliminares con el fin de descartar posibles fracasos debidos a factores individuales (por ejemplo, ejemplares demasiado jóvenes que no fructifican bien), ajustar técnicas y evaluar la factibilidad de las tareas a realizar considerando el esfuerzo de muestreo, ya que varias especies florecen juntas. Allophylus edulis, C. montevidense, $O$. acutifolia y $B$. salicifolius lo hacen en primavera, también las exóticas $(R$. ulmifolius y L. lucidum) pero su floración se extiende hasta comienzos del verano, y sólo $M$. laetevirens florece en verano (Montaldo, 2000). Por este motivo, cinco especies (A. edulis, B. salicifolius, $R$. ulmifolius, L. lucidum y M. laetevirens) se muestrearon en el año 2001 y las dos restantes $(C$. montevidense y $O$. acutifolia) en el siguiente año (2002). Ambos años de muestreo fueron similares en sus condiciones climáticas: temperatura media anual ${ }^{\circ} \mathrm{C} 18,8$ vs. 18,2 y precipitaciones $\mathrm{mm}$ 1749,7 vs. 1634,2 (datos del Observatorio Central Buenos Aires). En cada especie se seleccionaron tres individuos, donde se marcaron dos grupos de flores por individuo y tratamiento. Se realizaron en total 5 tratamientos: 1) testigos de polinización libre, 2) anemofilia (flores no manipuladas fueron embolsadas con tul, el cual deja pasar los granos de polen pero impide la entrada de insectos) y 3) autopolinización espontánea (flores no manipuladas fueron embolsadas con tela, la cual excluye tanto el paso del polen como la entrada de insectos; este tratamiento no permite diferenciar la ocurrencia de autopolinización automática de la apomixis, porque no se removieron las anteras). En seis de las especies (presumiblemente entomófilas, ver más adelante) se realizaron, además, tratamientos de polinización manual con pinceles, después de lo cual las flores fueron embolsadas con tela: 4) polinización cruzada manual (las flores fueron polinizadas utilizando polen de otro individuo), y 5) autopolinización manual (las flores fueron polinizadas con polen propio) en las especies con flores hermafroditas ( $R$. ulmifolius, L. lucidum, $B$. salicifolius y $C$. montevidense). Luego de la fructificación se contabilizó el número de frutos producidos y, como medida del éxito reproductivo se calculó el porcentaje de fructificación. Los datos utilizados en los análisis fueron los promedios por planta (i.e., individuo). En las especies con flores hermafroditas se determinó el índice de auto-compatibilidad, calculado mediante el cociente entre el éxito reproductivo de los tratamientos 5) 
autopolinización manual y 4) polinización cruzada manual. Si este índice fue menor a 0,2 se consideró a la especie como auto-incompatible (Morales \& Galetto, 2003 y referencias). Adicionalmente, en todas las especies estudiadas se calculó la relación polen-óvulo, un indicador del sistema reproductivo que presenta valores bajos en especies autógamas obligadas y elevados en las xenógamas ( $\overline{\mathrm{x}} \mathrm{ca} .30 \mathrm{vs}$. ca. 6000, Cruden, 1977). El número de granos de polen se determinó macerando androceos en glicerina $50 \%$, el recuento se hizo bajo microscopio utilizando un hemocitómetro (Kearns \& Inouye, 1993); para el número de óvulos se usó un estereoscopio $(\mathrm{N}=2-3$ individuos/especie y 10 flores/individuo).

\section{Cantidad y calidad del polen}

Para estudiar las cargas polínicas estigmáticas se coleccionaron inflorescencias de los individuos marcados. Se eligieron flores arbitrariamente (10 por individuo) y se recuperó el polen de los estigmas con etanol $70 \%$ en un portaobjetos excavado, revisando luego cada estigma para confirmar que todo el polen fue liberado (McLernon et al., 1996). El lavado con etanol permite desagregar los granos de polen y facilita el recuento (e.g., Dafni et al., 2005). La viabilidad del polen se determinó mediante la técnica de Greissl (1989), utilizando un microscopio de fluorescencia y ioduro de propidio y diacetato de fluoresceina como colorantes vitales. Con un objetivo de $10 \times$ se contaron los granos viables y no viables en 10 campos microscópicos de 4,95 $\mathrm{mm}^{2}$ (2 preparados por individuo con mezcla de al menos 3 flores). Se consideraron viables los granos germinados (largo del tubo polínico igual o mayor que el diámetro del grano, Abdul-Baki, 1992) y no germinados teñidos de verde, y no viables a los no germinados teñidos de rojo. También se contaron los granos de polen heteroespecíficos.

\section{Visitantes florales}

Para conocer los visitantes florales de las especies, se realizaron observaciones directas sobre las flores de los individuos controlados en los tratamientos de sistema reproductivo entre las 09:00 y 18:00 horas en días diferentes, con un promedio de 2 $\mathrm{h}$ de observación en cada una de las especies. Utilizando redes entomológicas se capturaron individuos representativos de los distintos insectos y se los sacrificó en el campo en frascos con cianuro de potasio. Luego, fueron montados siguiendo las técnicas de rutina y se los determinó en el laboratorio o con ayuda de especialistas (ver Agradecimientos). Con el fin de registrar los visitantes tan completamente como fuese posible se hicieron capturas también en el Jardín Botánico y parque de la Facultad de Agronomía de la Universidad de Buenos Aires ( $34^{\circ} 40^{\prime} \mathrm{S}, 58^{\circ} 30^{\prime} \mathrm{O}$ ) donde, con excepción de $R$. ulmifolius, existen individuos cultivados de las especies en estudio. Todos los ejemplares están depositados en la colección entomológica de la Cátedra de Botánica General de la misma facultad.

\section{Análisis de datos}

Se evaluó si existían diferencias significativas en la proporción de fructificación (éxito reproductivo) entre los tratamientos de sistema reproductivo mediante un análisis no paramétrico de la varianza (Kruskal-Wallis) y luego se hicieron comparaciones múltiples. Se consideró que una especie es preferentemente xenógama cuando se obtuvo mayor producción de frutos en las flores polinizadas con polen de otro individuo que del mismo individuo, que depende del viento (anemofilia) cuando la producción de frutos fue mayor en las flores embolsadas con tul que en las embolsadas con tela (ambas no manipuladas) y que está limitada polínicamente cuando en las flores polinizadas con polen de otro individuo se obtuvo mayor producción de frutos que en el control (Dafni et al., 2005). Para evaluar si existe alguna relación entre el grado de generalismo (i.e., número de especies de visitantes florales) de las plantas con su producción natural de frutos, y con la cantidad de polen heteroespecífico sobre el estigma de las flores, y entre la fructificación y la proporción de polen heteroespecífico en los estigmas, se realizaron análisis de correlación de Pearson. En función de alcanzar normalidad y homogeneidad de las varianzas, los datos fueron transformados utilizando el logaritmo natural para conteos y la trasformación angular o arco-seno en el caso de los porcentajes.

\section{Resultados}

Sistema reproductivo y calidad del servicio de polinización

La nativa $B$. salicifolius fue la única especie autocompatible y tuvo una producción de frutos de ca. $25 \%$ o superior para todos los 


\section{N. H. Montaldo et al. - Polinización en una selva invadida por plantas exóticas}

tratamientos. Cuando recibió manualmente polen de otro individuo (polinización cruzada manual) su fructificación se duplicó respecto a la obtenida bajo autopolinización manual y bajo polinización libre (Tabla 1). En las otras especies (nativas y exóticas) con flores hermafroditas el aporte de polen xenógamo incrementó el éxito reproductivo respecto a la polinización manual con polen autógamo, pero no respecto a la polinización libre. En el caso de la especie exótica L. lucidum, la producción de frutos de las flores polinizadas manualmente con polen xenógamo no difirió significativamente de la de las flores testigo, mientras que en $C$. montevidense (nativa) y en $R$. ulmifolius (exótica) fue significativamente menor.

Considerando las especies nativas dioicas, el aporte de polen a las flores femeninas aumentó significativamente la fructificación respecto a la polinización libre en $A$. edulis, pero no en $O$. acutifolia, donde la producción de frutos bajo polinización cruzada manual fué significativamente inferior a la de las flores testigo. Myrsine laetevirens produjo significativamente mayor cantidad de frutos en las flores pistiladas sometidas a exclusión parcial (anemofilia) que en las sometidas a exclusión total (autopolinización espontánea). Las flores pistiladas tienen estambres menos desarrollados que los de las estaminadas y contienen polen viable, aunque en mucho menor cantidad (ca. 6500 vs. ca. 69000 granos por flor).

Todas las especies de plantas (nativas y exóticas) tuvieron una relación polen-óvulo elevada (sólo en la exótica $R$. ulmifolius la relación polen-óvulo fue inferior al millar).

En condiciones naturales tres especies nativas ( $A$. edulis, B. salicifolius y $M$. laetevirens) recibieron menos granos de polen viable por estigma que óvulos posee la flor (Tabla 2).

El polen heteroespecífico en los estigmas de las especies estudiadas fue comparativamente escaso y sólo en la especie nativa $O$. acutifolia superó al número de óvulos (Tabla 2). La proporción de polen heteroespecífico encontrada en los estigmas no se asoció significativamente con la fructificación de las especies $(r=0,16 ; P=0,73 ; \mathrm{N}=7)$.

\section{Visitantes florales}

Las flores de seis especies fueron visitadas por insectos y las de $M$. laetevirens no recibieron visitantes. Se registraron 48 especies de insectos
(Tabla 3) pertenecientes a 13 familias y 4 órdenes: Diptera (39.58\%), Hymenoptera (39.58\%), Lepidoptera $(14.58 \%)$ y Coleoptera $(6.25 \%)$. El único visitante común a todas las plantas fue la abeja doméstica (Apis mellifera). Otros 6 visitantes fueron compartidos por las especies nativas y las exóticas, 3 de ellos en Punta Lara. El número de especies visitantes varió entre 9, en $O$. acutifolia, y 21 en $B$. salicifolius. No se halló una asociación significativa en entre el grado de generalismo de las plantas y la fructificación de las mismas en Punta Lara ( $r=$ -0,25; $P=0,64 ; \mathrm{N}=6$ ). De hecho, por ejemplo $A$. edulis (la especie de peor perfomance reproductiva, Tabla 1) fue visitada por 11 especies de insectos y C. montevidense (la de mayor fructificación) sólo por 5. El grado de generalismo tampoco se asoció de manera significativa con la cantidad de polen heteroespecífico $(r=0,31 ; P=0,55 ; \mathrm{N}$ =6). Los elencos de A. edulis, B. salicifolius, $C$. montevidense y $L$. lucidum estuvieron dominados por Dípteros e Himenópteros (Fig. 1). En $R$. ulmifolius además de los Himenópteros fueron importantes los Lepidópteros, mientras que $O$. acutifolia fue visitada casi exclusivamente por Dípteros.

\section{Discusión}

Sistema reproductivo y visitantes florales

Todas las especies de plantas (nativas y exóticas) tuvieron una relación polen-óvulo compatible con la conocida para especies xenógamas. Los resultados coincidentes de los experimentos manipulativos para conocer el sistema reproductivo de las plantas y sus relaciones polen-óvulo indican que necesitan agentes polinizadores para formar frutos y semillas. Si bien la relación polen óvulo de la especie exótica $R$. ulmifolius se aproxima al de una especie xenógama facultativa ( $\bar{x}$ ca. 800, Cruden, 1977), a diferencia de lo esperado (Rambuda \& Johnson, 2004; Hao et al., 2011) la única especie que se comportó como autocompatible fue la nativa $B$. salicifolius.

Seis de las especies (4 nativas y 2 exóticas) son polinizadas por insectos mientras que $M$. laetevirens (nativa) es anemófila, dada la ausencia de visitantes florales y los resultados obtenidos en los tratamientos para conocer el sistema 
Bol. Soc. Argent. Bot. 52 (4) 2017

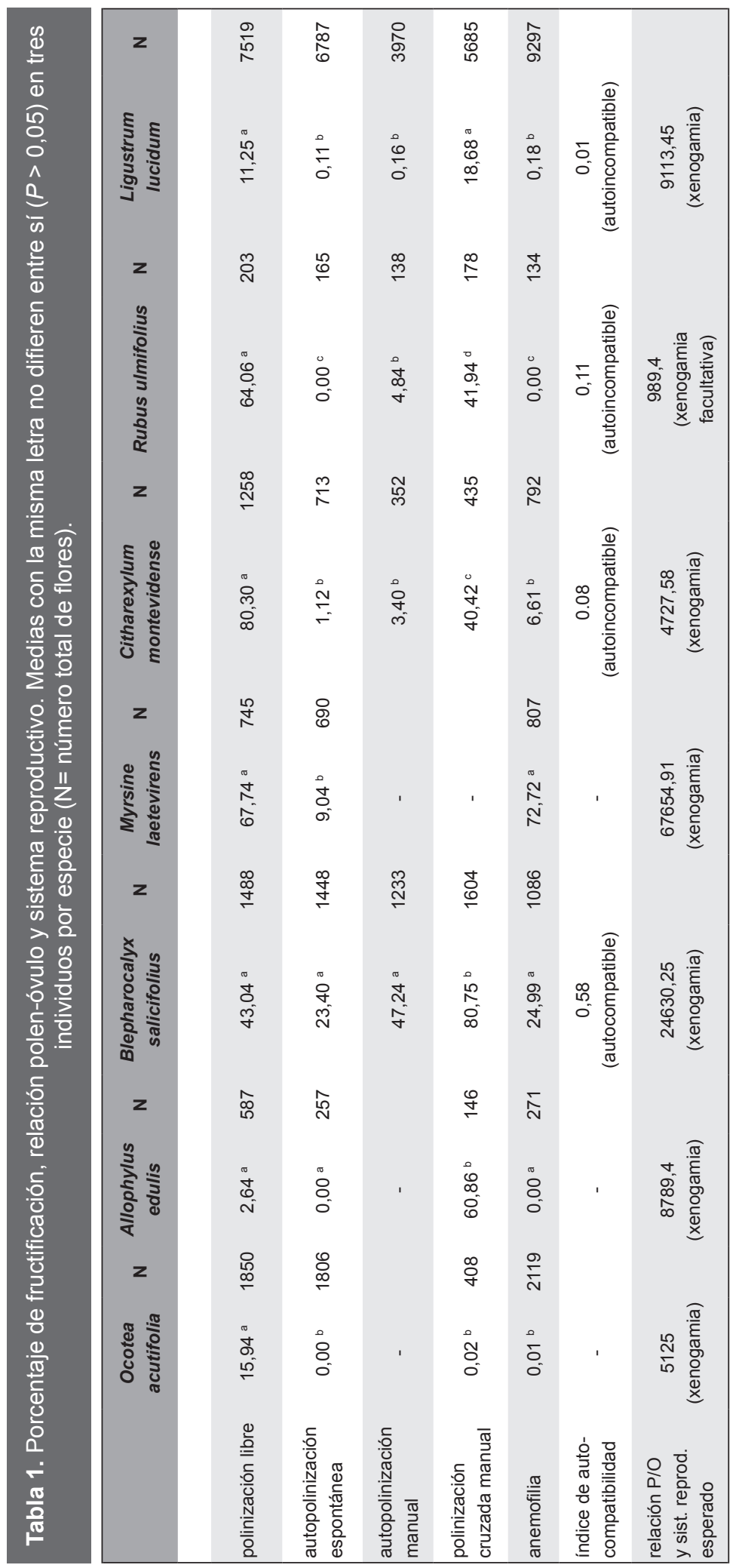


Tabla 2. Número medio de óvulos por flor, cantidad de polen conespecífico y heteroespecífico en las cargas estigmáticas (CE) y viabilidad del polen.

\begin{tabular}{|lcccccccc|}
\hline & $\begin{array}{c}\text { Ocotea } \\
\text { acutifolia }\end{array}$ & $\begin{array}{c}\text { Allophylus } \\
\text { edulis }\end{array}$ & $\begin{array}{c}\text { Blepharocalyx } \\
\text { salicifolius }\end{array}$ & $\begin{array}{c}\text { Myrsine } \\
\text { laetevirens }\end{array}$ & $\begin{array}{c}\text { Citharexylum } \\
\text { montevidense }\end{array}$ & $\begin{array}{c}\text { Rubus } \\
\text { ulmifolius }\end{array}$ & $\begin{array}{c}\text { Ligustrum } \\
\text { lucidum }\end{array}$ \\
\hline óvulos por flor & 1 & 2,16 & 31,15 & 2 & 3,7 & 72 & 3,33 \\
$\begin{array}{l}\text { granos de polen } \\
\text { propio por CE }\end{array}$ & 20,97 & 2,9 & 30,67 & 1,7 & 7,22 & 580,16 & 33,92 \\
$\begin{array}{l}\text { granos de polen } \\
\text { extraño por CE }\end{array}$ & 2,7 & 0,35 & 2,39 & 1,5 & 0,54 & 6,66 & 1,46 \\
$\begin{array}{l}\text { polen } \\
\text { heteroespe- } \\
\text { cífico (\%) }\end{array}$ & 11,41 & 10,77 & 7,12 & 46,87 & 6,96 & 1,13 & 4,13 \\
$\begin{array}{l}\text { granos de polen } \\
\text { propio por óvulo }\end{array}$ & 20,97 & 1,34 & 0,98 & 0,85 & 1,95 & 8,06 & 10,19 \\
$\begin{array}{l}\text { viabilidad del } \\
\text { polen (\%) }\end{array}$ & 42,86 & 59,08 & 64,42 & 59,86 & 86,24 & 92,95 & 30,58 \\
$\begin{array}{l}\text { granos viables } \\
\text { por óvulo }\end{array}$ & 8,99 & 0,79 & 0,63 & 0,5 & 1,68 & 7,49 & 3,11 \\
\hline
\end{tabular}

reproductivo (anemofilia vs. polinización libre y anemofilia vs. autopolinización espontánea). Considerando las plantas entomófilas, una de las exóticas (L. lucidum) compartió con las nativas (A. edulis, B. salicifolius, C. montevidense) los grupos funcionales dominantes de visitantes florales (Dípteros e Himenópteros) y la otra exótica ( $R$. ulmifolius) fue la que recibió más especies de Lepidópteros. Otegui \& Cocucci (1999) ya habían comprobado en Punta Lara que $M$. laetevirens depende del viento para la polinización. No observaron producción de frutos en las flores pistiladas cubiertas con bolsas de papel ni encontraron granos de polen en sus anteras, por lo que consideraron a la especie dioica. La fructificación registrada para el tratamiento de autopolinización espontánea en el presente estudio podría indicar presencia de autogamia o de apomixis (mencionada en la familia Myrsinaceae para Ardisia, Asker \& Jerling, 1992). La primera alternativa es más probable dado que en nuestro estudio sí se halló polen viable en las anteras, que son dehiscentes (Otegui \& Cocucci, 1999).
Calidad del servicio de polinización y limitación por polen

Los resultados sugieren que en Punta Lara hay especies nativas que enfrentan limitación por polen, porque $A$. edulis y $B$. salicifolius produjeron menos frutos en condiciones de polinización natural que cuando se agregó polen de otro individuo (polinización cruzada manual), demostrando que la falta de polen compatible limita su reproducción (Burd, 1994; Dafni et al., 2005; Knight et al., 2005; Aizen \& Harder, 2007). Aunque en el presente estudio no se realizó el tratamiento de polinización cruzada manual en M. laetevirens, Otegui \& Cocucci (1999) también encontraron limitación polínica para esta especie. La falta de respuesta de las restantes especies a este tratamiento podría deberse a que no están limitadas por polen, no pudiendo descartarse errores o defectos asociados al mismo (Young \& Young 1992). Además, si se considera que para alcanzar el máximo de producción de semillas es necesario un mínimo de 4-6 granos de polen por óvulo (Cruden, 2000), sólo una de las nativas ( $O$. acutifolia) y las dos exóticas se aproximan a esta condición (Tabla 2). Las especies dioicas como $A$. 
Tabla 3. Visitantes florales (especie, Orden, Familia) de las especies de plantas estudiadas. $1=$ presencia en la selva ribereña de Punta Lara, 2 = presencia en la Facultad de Agronomía de la Universidad de Buenos Aires. En Myrsine laetevirens no se observaron visitantes florales.

\begin{tabular}{|c|c|c|c|c|c|c|c|c|}
\hline Especie visitante & Orden & Familia & $\begin{array}{c}\text { Ocotea } \\
\text { acutifolia }\end{array}$ & $\begin{array}{l}\text { Allophylus } \\
\text { edulis }\end{array}$ & $\begin{array}{c}\text { Blepharocalyx } \\
\text { salicifolius }\end{array}$ & $\begin{array}{l}\text { Citharexylum } \\
\text { montevidense }\end{array}$ & $\begin{array}{l}\text { Rubus } \\
\text { ulmifolius }\end{array}$ & $\begin{array}{l}\text { Ligustrum } \\
\text { lucidum }\end{array}$ \\
\hline Compsocerus sp. & Coleoptera & Cerambycidae & & & 1 & & & \\
\hline Megacylene sp. & Coleoptera & Cerambycidae & & & & & & 1 \\
\hline Sp. no identificada & Coleoptera & Meloidae? & & & 1 & & & \\
\hline Phaenicia cluvia & Diptera & Calliphoridae & 1 & 2 & & & & \\
\hline Sp. no identificada & Diptera & Muscidae & & 1 & & & & \\
\hline Allograpta exotica & Diptera & Syrphidae & 2 & 1 & 1 & & & \\
\hline Allograpta sp. & Diptera & Syrphidae & 1 & & & & & \\
\hline Carposcalis saltana & Diptera & Syrphidae & 2 & & & 2 & & \\
\hline $\begin{array}{l}\text { Carposcalis } \\
\text { punctulata }\end{array}$ & Diptera & Syrphidae & & 2 & & & & \\
\hline Copestylum n. sp. & Diptera & Syrphidae & & & 1 & 1 & & 1 \\
\hline Eristalinus taeniops & Diptera & Syrphidae & & & & & & 1.2 \\
\hline $\begin{array}{l}\text { Ocyptamus } \\
\text { fuscicosta }\end{array}$ & Diptera & Syrphidae & & & 1 & & & \\
\hline $\begin{array}{l}\text { Ocyptamus } \\
\text { meridionalis }\end{array}$ & Diptera & Syrphidae & 2 & & 1.2 & & & \\
\hline Ocyptamus obliquus & Diptera & Syrphidae & & & 1 & & & \\
\hline Ocyptamus sp. & Diptera & Syrphidae & & 2 & & & & \\
\hline $\begin{array}{l}\text { Palpada } \\
\text { distinguenda }\end{array}$ & Diptera & Syrphidae & & & & 1.2 & & 2 \\
\hline Palpada furcata & Diptera & Syrphidae & & & 2 & & & 2 \\
\hline Palpada rufiventris & Diptera & Syrphidae & & 1 & 1 & & 1 & 1 \\
\hline Syrphus reedi & Diptera & Syrphidae & 1 & 1.2 & 1.2 & 1 & & 2 \\
\hline Toxomerus sp. & Diptera & Syrphidae & 1 & & & 2 & & \\
\hline Trafoia sp. & Diptera & Tachinidae & 1 & & & & & \\
\hline Sp. no identificada & Diptera & Tachinidae & & & & & 1 & \\
\hline Apis mellifera & Hymenoptera & Apidae & 1 & 1.2 & 1 & 1 & 1 & 1 \\
\hline Bombus atratus & Hymenoptera & Apidae & & & 1.2 & & 1 & \\
\hline $\begin{array}{l}\text { Melissodes } \\
\text { tintinnans }\end{array}$ & Hymenoptera & Apidae & & & & & 1 & 1 \\
\hline Xylocopa augusti & Hymenoptera & Apidae & & & & & & 1 \\
\hline Plebeia sp. & Hymenoptera & Halictidae & & 1.2 & 2 & & & \\
\hline $\begin{array}{l}\text { Augochloropsis } \\
\text { tupacamaru }\end{array}$ & Hymenoptera & Halictidae & & 1 & 2 & & & \\
\hline $\begin{array}{l}\text { Augochloropsis } \\
\text { multiplex }\end{array}$ & Hymenoptera & Halictidae & & & & & 1 & \\
\hline $\begin{array}{l}\text { Augochlora } \\
\text { amphitrite }\end{array}$ & Hymenoptera & Halictidae & & & & 1 & 1 & \\
\hline $\begin{array}{l}\text { Augochlora } \\
\text { nausicaa }\end{array}$ & Hymenoptera & Halictidae & & 1 & & & & \\
\hline Lasioglossum sp. & Hymenoptera & Halictidae & & & & & & 2 \\
\hline $\begin{array}{l}\text { Anthodioctes } \\
\text { megachiloides }\end{array}$ & Hymenoptera & Megachilidae & & & 2 & 2 & & 1 \\
\hline
\end{tabular}




\begin{tabular}{|c|c|c|c|c|c|c|c|c|}
\hline Especie visitante & Orden & Familia & $\begin{array}{c}\text { Ocotea } \\
\text { acutifolia }\end{array}$ & $\begin{array}{l}\text { Allophylus } \\
\text { edulis }\end{array}$ & $\begin{array}{l}\text { Blepharocalyx } \\
\text { salicifolius }\end{array}$ & $\begin{array}{l}\text { Citharexylum } \\
\text { montevidense }\end{array}$ & $\begin{array}{l}\text { Rubus } \\
\text { ulmifolius }\end{array}$ & $\begin{array}{l}\text { Ligustrum } \\
\text { lucidum }\end{array}$ \\
\hline $\begin{array}{l}\text { Megachile } \\
\text { (Cressoniella) sp. }\end{array}$ & Hymenoptera & Megachilidae & & & & 2 & & \\
\hline $\begin{array}{l}\text { Megachile } \\
\text { (Leptorachis) sp. }\end{array}$ & Hymenoptera & Megachilidae & & & 2 & & & \\
\hline $\begin{array}{l}\text { Megachile } \\
\text { (Pseudocentron) sp. }\end{array}$ & Hymenoptera & Megachilidae & & & 2 & & & \\
\hline Hylaeus punctatus & Hymenoptera & Colletidae & & & 2 & & & \\
\hline Hylaeus sp. & Hymenoptera & Colletidae & & 1 & 2 & & & \\
\hline $\begin{array}{l}\text { Brachygastra } \\
\text { lecheguana }\end{array}$ & Hymenoptera & Vespidae & & 1 & 1.2 & & & \\
\hline Polistes sp. & Hymenoptera & Vespidae & & & & & 1 & \\
\hline Polybia scutellaris & Hymenoptera & Vespidae & & 1 & 1.2 & & & \\
\hline Epargyreus tmolis & Lepidoptera & Hesperiidae & & & & & 1 & \\
\hline Sp. no identificada 1 & Lepidoptera & Hesperiidae & & & & & 1 & \\
\hline Sp. no identificada 2 & Lepidoptera & Hesperiidae & & & & 2 & & \\
\hline Actinote pellenea & Lepidoptera & Nymphalidae & & & & 2 & & \\
\hline Ortilia ithra & Lepidoptera & Nymphalidae & & & & & 1 & \\
\hline Ortilia velica & Lepidoptera & Nymphalidae & & & & & & 1 \\
\hline Tegosa claudina & Lepidoptera & Nymphalidae & & & & & 1 & \\
\hline $\begin{array}{l}\text { Especies en } \\
\text { Punta Lara }\end{array}$ & & & 6 & 11 & 13 & 5 & 12 & 9 \\
\hline Especies totales & & & 9 & 14 & 21 & 11 & 12 & 13 \\
\hline
\end{tabular}

edulis (fue la de menor porcentaje de fructificación) serían las más afectadas, pero también algunas de las que tienen flores monoclinas sufren déficit de polen (e.g. B. salicifolius) y no pueden alcanzar su máximo potencial reproductivo, porque las flores reciben menos polen compatible que el requerido. $\mathrm{Al}$ parecer los agentes polinizadores no transfieren suficiente polen propio para fertilizar todos los óvulos disponibles en la población (Brunet, 2005; Waser \& Price, 2016).

La cantidad y proporción de polen heteroespecífico encontradas no influyeron en la fructificación de las especies ni tuvieron relación con la complejidad de los elencos de visitantes de las plantas. Los resultados obtenidos permiten descartar interferencias que signifiquen pérdidas reproductivas por oclusión de estigmas por los granos extraños (McLernon et al., 1996; Brown \& Mitchell, 2001) o causadas luego de la germinación de los mismos (Nishida et al., 2014).
La limitación por polen se evidencia tanto en especies visitadas por insectos como en la anemófila M. laetevirens. En las primeras no parece atribuible a la existencia de mutualismos altamente especializados. Si bien no se halló relación entre la fructificación natural y el número de especies de visitantes florales, son plantas generalistas a las que acuden conjuntos de insectos numerosos y taxonómicamente diversos, como es común en la mayoría de las plantas entomófilas (Gómez, 2002; Herrera, 2005). Una alta diversidad de visitantes no significa necesariamente menor limitación por polen (Davila et al., 2012), y puede existir un componente cualitativo tan importante como el cuantitativo (Aizen \& Harder, 2007).

\section{Situación actual y futuros estudios}

La supervivencia de una especie de planta puede evaluarse considerando la importancia que tienen las semillas en su demografía, la probabilidad 


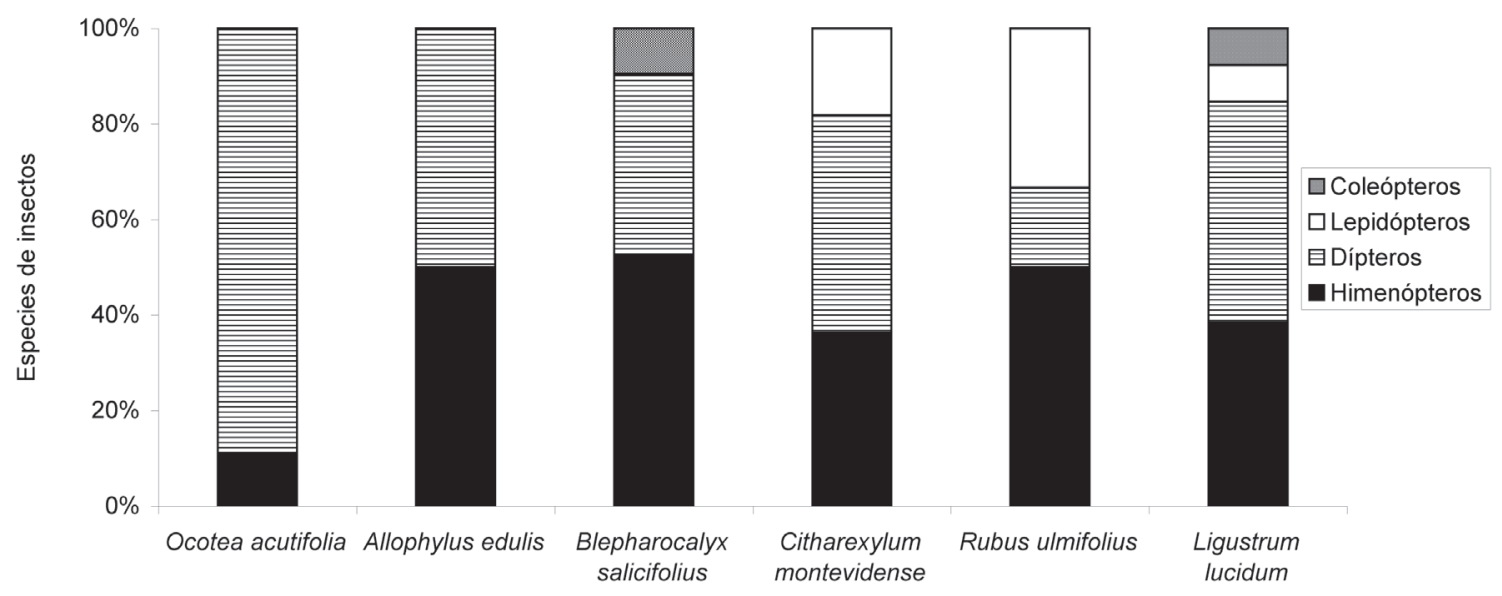

Fig. 1. Composición relativa de los elencos de visitantes florales. Se incluyen todos los visitantes florales (selva ribereña de Punta Lara y Facultad de Agronomía de la Universidad de Buenos Aires).

del fracaso de los procesos de polinización o de dispersión y la dependencia reproductiva en los mutualismos (Bond, 1944). Este trabajo aporta información sobre el sistema reproductivo de las plantas nativas (son xenógamas y dependen de sus semillas para perpetuarse en la selva) y de las exóticas invasoras, sobre los agentes polinizadores que intervienen en cada caso, y sugiere que para varias de las nativas el servicio de polinización es insuficiente.

La introducción de L. lucidum produjo un gran cambio demográfico en la selva porque compite con éxito con el resto de las plantas, a las que va reemplazando. En poco más de cincuenta años la abundancia relativa de sus individuos reproductivos aumentó del 1,85\% al 50\%, mientras que la del conjunto de las nativas evolucionó en forma inversa (Montaldo, 2000). En estudios futuros debería evaluarse si la reducción de las poblaciones de las especies nativas, asociada al avance de L. lucidum, y la presencia de las exóticas, que comparten algunos visitantes con las nativas (Tabla 1), son causantes de limitación por polen más allá de los niveles que son naturales para estas especies. La limitación por polen es un fenómeno frecuente y generalizado en la naturaleza (e.g. Burd, 1994; Knight et al., 2005) y refleja causas diferentes y no siempre bien determinadas (Ashman et al.; 2004). Sin embargo, su magnitud puede aumentar en presencia de disturbios humanos (Aizen \& Feinsinger, 1994; Ashman et al.; 2004; Knight et al., 2005; Aguilar et al., 2006), la introducción de plantas exóticas produce en general un efecto negativo sobre la reproducción de las nativas (Chittka \& Schürken, 2001; Morales \& Traveset, 2009), mayor a medida que aumenta la densidad de aquellas (Traveset, 2015). Además del hábitat en que crecen las plantas sería de interés analizar en forma más exhaustiva los elencos de visitantes florales y sus cargas polínicas, ya que en la limitación por polen influye también la efectividad de los mismos. (Davila et al., 2012). La información disponible indica que son elencos diversos pero no permite descartar falta de polinizadores.

\section{Agradecimientos}

En los trabajos de campo participó M.V. Vasellati, que además colaboró ampliamente en las tareas de laboratorio. A. Roig Alsina (Hymenoptera), F.C. Thompson (Syrphidae), A. Bachman (Coleoptera), S. Durante (Megachilidae), D. Medan y J.P Torretta colaboraron en la determinación de visitantes florales y N.H. Bartoloni en el análisis estadístico. D. Medan aportó valiosas opiniones durante el transcurso del trabajo y con la lectura del manuscrito. Los comentarios y sugerencias de dos revisores anónimos contribuyeron a mejorarlo. 


\section{N. H. Montaldo et al. - Polinización en una selva invadida por plantas exóticas}

\section{Biblografía}

ABDUL-BAKI, A. A. 1992. Determination of pollen viability in Tomatoes. J. Amer. Soc. Hort. Sci. 117: 473-476.

AGUILAR, R., L. ASHWORTH, L. GALETTO \& M. A. AIZEN. 2006. Plant reproductive susceptibility to habitat fragmentation: Review and synthesis through a meta-analysis. Ecol. Lett. 9: 968-980.

AIZEN, M. A. 2007. Enfoques en el estudio de la reproducción sexual de las plantas en ambientes alterados: limitaciones y perspectivas. Ecología Austral 17: 7-19.

AIZEN, M. A. \& P. FEINSINGER. 1994. Forest fragmentation, pollination, and plant reproduction in a Chaco dry forest, Argentina. Ecology 75: 330-351.

AIZEN, M. A. \& L. D. HARDER. 2007. Expanding the limits of the pollen limitation concept: effects of pollen quantity and quality. Ecology 88: 271-281.

AIZEN, M. A., C. L. MORALES \& J. M. MORALES. 2008. Invasive mutualists erode native pollination webs. PLoS Biol. 6: 396-403.

ASHMAN, T.-L., T. M. KNIGHT, J. A. STEETS, P. AMARASEKARE, M. BURD, D. R. CAMPBELL, M. R. DUDASH, M. O. JOHNSTON, S. J. MAZER, R. J. MITCHELL, M. T. MORGAN \& W. G. WILSON. 2004. Pollen limitation of plant reproduction: Ecological and evolutionary causes and consequences. Ecology 85: 2408-2421.

ASKER, S. \& L. JERLING. 1992. Apomixis in plants. CRC Press Inc., Boca Raton, Florida.

BELL, J. M., J. D. KARRON \& R. J. MITCHELL. 2005. Interspecific competition for pollination lowers seed production and outcrossing rate in Mimulus ringens. Ecology 86: 762-771.

BJERKNES, A. L., Ø. TOTLAND, S. J. HEGLAND \& A. NIELSEN. 2007. Do alien plant invasions really affect pollination success in native plant species? Biol. Conserv. 138: 1-12.

BOND, W. J. 1994. Do mutualisms matter? - Assessing the impact of pollinator and disperser disruption on plant extinction. Philos. T. Roy. Soc. B 344: 83-90.

BROWN, B. J. \& R. J. MITCHELL. 2001. Competition for pollination: effects of pollen of an invasive plant on seed set of a native congener. Oecologia 129: 43-49.

BRUNET, J. 2005. Plant-pollinator interactions and pollen dispersal. In: DAFNI, A., P. G. KEVAN \& B. C. HUSBAND (eds.), Practical Pollination Biology, pp. 56-82. Enviroquest, Ltd., Cambridge, Ontario.

BURD, M. 1994. Bateman's principle and plant reproduction: the role of pollen limitation in fruit and seed set. Bot. Rev. 60: 83-139.
CABRERA, A. L. 1960. La selva marginal de Punta Lara. Ciencia e Investigación 16: 439-446.

CABRERA, A. L. 1975. Regiones fitogeográficas argentinas. Enciclopedia argentina de agricultura y jardinería. Tomo 2, Fascículo 1. ACME, Buenos Aires.

CABRERA, A. L. \& G DAWSON. 1944. La selva marginal de Punta Lara en la ribera argentina del Río de la Plata. Rev. Mus. La Plata 5: 267-382.

CHITTKA, L. \& S. SCHÜRKENS. 2001. Successful invasion of a floral market. Nature 411: 653.

CRUDEN, R. W. 1977. Pollen ovule ratios: a conservative indicator of breeding systems in flowering plants. Evolution 31: 32-44.

CRUDEN, R. W. 2000. Pollen grains: why so many? Plant Syst. Evol. 222: 143-165.

DAFNI, A., E. PACCINI \& M. NEPPI. 2005. Pollen and stigma biology. In: DAFNI, A., P. G. KEVAN \& B C. HUSBAND (eds.), Practical Pollination Biology, pp. 83-146. Enviroquest, Ltd. Cambridge, Ontario.

DAVILA, Y. C., E. ELlE, J. C. VAMOSI, L. HERMANUTZ, J. T. KERR, C. J. LORTIE, A. R. WESTWOOD, T. S. WOODCOCK \& A. C. WORLEY. 2012. Ecosystem services of pollinator diversity: a review of the relationship with pollen limitation of plant reproduction. Botany 90: 535543.

ELTON, C. S. 1958. The ecology of invasions by animals and plants. Methuen, London.

GÓMEZ, J. M. 2002. Generalización en las interacciones entre plantas y polinizadores. Rev. Chil. Hist. Nat. 75: 105-116.

GREISSL, R.1989. Vitality analysis of monadic and polyadic pollen grains using optical contrastfluorescence microscopy. Sci. Tech. Inform. 11: 180-184.

GUERRERO, E. L. 2014. El paradigma del relicto de selva marginal y las políticas de conservación de bosques nativos en el noreste de la provincia de Buenos Aires: una revisión crítica. IPID, Buenos Aires. DOI: 10.13140/2.1.3261.3444.

HAO, J. H., S. QIANG, T. CHROBOCK, M. VAN KLEUNEN \& Q. Q. LIU. 2011. A test of baker's law: breeding systems of invasive species of Asteraceae in China. Biol. Invasions 13: 571-580.

HERRERA, C. M. 2005. Plant generalization on pollinators: species property or local phenomenon. Am. J. Bot. 92: 13-20.

KAISER-BUNBURY, C. N. \& C. B. MÜLLER. 2009. Indirect interactions between invasive and native plants via pollinators. Naturwissenschaften 96: 339346.

KEARNS, C. A. \& D. W. INOUYE. 1993. Techniques for pollination biologists. University Press of Colorado, Boulder, Colorado. 
KEARNS, C. A., D. W. INOUYE \& N. M. WASER. 1998. Endangered mutualism: the conservation of plant-pollinator interactions. Annu. Rev. Ecol. Evol. Syst. 29: 83-112.

KNIGHT, T. M., J. A. STEETS, J. C. VAMOSI, S. J. MAZER, M. BURD, D. R. CAMPBELL, M. R. DUDASH, M. O. JOHNSTON, R. J. MITCHELL \& T. L. ASHMAN. 2005. Pollen limitation of plant reproduction: pattern and process. Annu. Rev. Ecol. Evol. Syst. 36: 467-97.

MATSUMOTO, T., K. TAKAKURA \& N. TAKAYOSHI. 2010. Alien pollen grains interfere with the reproductive success of native congener. Biol. Invasions 12: 1617-1626.

MATSUMOTO, T., K. TAKAKURA \& T. NISHIDA. 2011. Alien Dandelion displace a native related species through interspecific pollen transfer. In: YSAGI Y. \& Y. SUYAMA (eds), Single-pollen genotyping, pp. 83-93. Ecological Research Monographs. Springer, Tokyo.

MEMMOTT, J. \& N. M. WASER. 2002. Integration of aliens plants into a native flower-pollinator visitation web. P. Roy. Soc. Lond. B Bio. 269: $2395-$ 2399.

MCLERNON, S. M., S. D. MURPHY \& L. W. AARSEN. 1996. Heterospecific pollen transfer between sympatric species in a midsuccesional old-field community. Am. J. Bot. 83: 1168-1174.

MONTALDO, N. H. 1993. Dispersión por aves y éxito reproductivo de dos especies de Ligustrum (Oleaceae) en un relicto de selva subtropical en la Argentina. Rev. Chil. Hist. Nat. 66: 75-85.

MONTALDO, N. H. 2000. Éxito reproductivo de plantas ornitócoras en un relicto de selva subtropical en la Argentina. Rev. Chil. Hist. Nat. 73: 511-524 (corrigendum en Rev. Chil. Hist. Nat. 73: 831, 2000).

MONTALDO, N. H. 2005. Aves frugívoras de un relicto de selva subtropical ribereña en Argentina: manipulación de frutos y destino de las semillas. Hornero 20: 163-172.

MORAGUES, E. \& A. TRAVESET. 2005. Effect of Carpobrotus spp. on the pollination success of native plant species of the Balearic Islands. Biol. Conserv. 122: 611-619.

MORALES, C. L. \& L. GALETTO. 2003. Influence of compatibility system and life form on plant reproductive success. Plant Biol. 5: 567-573.

MORALES, C. L. \& M. A. AIZEN. 2006. Invasive mutualisms and the structure of plant-pollinator interactions in the temperate forests of north-west Patagonia, Argentina. J. Ecol. 94: 171-180.

MORALES, C. L. \& A. TRAVESET. 2009. A metaanalysis of impacts of alien vs. native plants on pollinator visitation and reproductive success of co-flowering native plants. Ecol. Lett. 12: 716-728.
NISHIDA, N., M. M. KANAOKA, K. HASHIMOTO, K.-I. TAKAKURA \& T. NISHIDA. 2014. Pollenpistil interactions in reproductive interference: comparisons of heterospecific pollen tube growth from alien species between two native Taraxacum species. Funct. Ecol. 28: 450-457.

NOBLE, I. R. 1989. Attributes of invaders and the invading process: terrestrial and vascular plants. In: DRAKE J. A., H. A. MOONEY, F. DI CASTRI, R. H. GROVES, F. J. KRUGER, M. REJMÁNEK \& M. WILLIAMSON (eds.), Biological invasions. A global perspective, pp. 301-313. SCOPE 37. J. Wiley \& Sons, Chichester.

OLLERTON, J., R. WINFREE \& S. TARRANT. 2011. How many flowering plants are pollinated by animals? Oikos 120: 321-326.

OTEGUI, M. \& A. COCUCCI. 1999. Flower morphology and biology of Myrsine laetevirens, structural and evolutionary implications of anemophily in Myrsinaceae. Nord. J. Bot. 19: 71-85.

RAMBUDA, T. D. \& S. D. JOHNSON. 2004. Breeding systems of invasive alien plants in South Africa: does Baker's rule apply? Divers. Distrib. 10: 409416.

RATHCKE, B. 1983. Competition and facilitation among plants for pollination. In: REAL L. (ed.), Pollination Biology, pp. 305-329. Academic Press, New York, NY.

REJMÁNEK, M. 1989. Invasibility of plant communities. In: DRAKE J. A., H. A. MOONEY, F. DI CASTRI, R. H. GROVES, F. J. KRUGER, M. REJMÁNEK \& M. WILLIAMSON (eds.), pp. 369-388, Biological invasions. A global perspective. SCOPE 37. J. Wiley \& Sons, Chichester.

RICHARDSON, D. M., N. ALLSOPP, C. M. D’ANTONIO, S. J. MILTON \& M. REJMÁNEK. 2000. Plant invasions - the role of mutualisms. Biol. Rev. 75: 65-93.

RICHARDSON, D. M. \& M. REJMÁNEK. 2011. Trees and shrubs as invasive alien species - a global review. Divers. Distrib. 17: 788-809.

SPIRA, T. P. 2001. Plant-pollinator interactions: a threatened mutualism with implications for the ecology and management of rare plants. Nat. Area. J. 21: 78-88.

TAKAKURA, K. \& S. FUJII. 2010. Reproductive interference and salinity tolerance differentiate habitat use between two alien cockleburs: Xanthium occidentale and $X$. italicum (Compositae). Plant Ecol. 206: 309-319.

TOTLAND, O., A. NIELSEN, A. L. BJERKNES \& M. OHLSON. 2006. Effects of an exotic plant and habitat disturbance on pollinator visitation and reproduction in a boreal forest herb. Am. J. Bot. 93: 868-873. 


\section{N. H. Montaldo et al. - Polinización en una selva invadida por plantas exóticas}

TRAVESET, A. \& D. M. RICHARDSON. 2006. Biological invasions as disruptors of plant reproductive mutualisms. Trends Ecol. Evol. 21: 208-216.

TRAVESET, A. \& D. M. RICHARDSON. 2014. Mutualistics interactions and biological invasions. Annu. Rev. Ecol. Evol. Syst. 45: 89-113.

TRAVESET, A. 2015. Impacto de las especies exóticas sobre las comunidades mediado por interacciones mutualistas. Ecosistemas 24: 67-75.

VAN DYKE, F. 2008. Conservation Biology: Foundations, Concepts, Applications. Second Edition. Springer, Dordrecht.

VILA, M., I. BARTOMEUS, A. C. DIETZSCH, T. PETANIDOU, I. STEFFAN-DEWENTER, J. C. STOUT \& T. TSCHEULIN. 2009. Invasive plant integration into native plant-pollinator networks across Europe. P. Roy. Soc. Lond. B Bio. 276: $3887-$ 3893.

VITOUSEK, P. M., C. M. DANTONIO; L. L. LOOPE; M. REJMANEK \& R. WESTBROOKS. 1997. Introduced species: A significant component of human-caused global change. New Zeal. J. Ecol. 21: 1-16.
YOUNG, H. J. \& T. P. YOUNG. 1992. Alternative outcomes of natural and experimental high pollen loads. Ecology 73: 640-647.

WASER, N. M. 1983. Competition for pollination and floral character differences among sympatric plant species: a review of evidence. In: JONES C. E. \& R. J. LITTLE (eds.), pp. 277-293, Handbook of Experimental Pollination Biology. Van Nostrand Reinhold, New York, NY.

WASER, N. M. \& M. W. PRICE. 2016. Drought, pollen and nectar availability, and pollination success. Ecology 97: 1400-1409.

Recibido el 27 de diciembre de 2016, aceptado el 25 de agosto de 2017. 
